\title{
Relational Complexes
}

\author{
Joop Leo
}

Received: 24 February 2011 / Accepted: 22 February 2012 / Published online: 27 April 2012 (C) The Author(s) 2012. This article is published with open access at Springerlink.com

\begin{abstract}
A theory of relations is presented that provides a detailed account of the logical structure of relational complexes. The theory draws a sharp distinction between relational complexes and relational states. A salient difference is that relational complexes belong to exactly one relation, whereas relational states may be shared by different relations. Relational complexes are conceived as structured perspectives on states 'out there' in reality. It is argued that only relational complexes have occurrences of objects, and that different complexes of the same relation may correspond to the same state.
\end{abstract}

Keywords Relational complex $\cdot$ Relational state $\cdot$ Substitution

\section{Introduction}

Many of us will consider Willem-Alexander's loving Máxima as a fact-as a true state 'out there' in the world. Many of us will also agree that this state can be broken up into parts: a binary relation with Willem-Alexander and Máxima as relata. But we can also conceive of this state as being composed of a unary relation (the property of loving Máxima) and Willem-Alexander as relatum. Is there a principled reason to prefer one analysis above the other? Going a step further, we can pose the fundamental question: Do states 'out there' have in any genuine sense a unique underlying relation and a unique set of relata?

According to Bertrand Russell in 'The Principles of Mathematics', a whole that has more than two parts may be analyzed in a plurality of ways, but

J. Leo $(\bowtie)$

Department of Philosophy, Utrecht University, Utrecht, The Netherlands

e-mail: joop.leo@phil.uu.nl 
two wholes composed of different terms must be different when all terms are simple [22, p. 77]. And later, in 'Theory of Knowledge', he writes:

A 'complex' is anything analyzable, anything which has constituents. [...] It may be questioned whether a complex is or is not the same as a 'fact', where a 'fact' may be described as what there is when a judgement is true, but not when it is false. [...] However this may be, there is certainly a oneone correspondence of complexes and facts, and for our present purposes we shall assume that they are identical [23, pp. 79-80].

In his paper 'Neutral Relations', Kit Fine expresses a similar view. He refers to states and facts as complexes, and writes: "We wish to adopt a conception of relations and their completions for which Uniqueness holds", where Uniqueness is the assumption that "no complex is the completion of two distinct relations" [7, pp.4-5].

What makes a view that identifies states 'out there' with relational complexes attractive is its apparent simplicity. I think this view suffers from serious problems, however, when combined with Uniqueness. In many cases it is not clear what the underlying relation - if any-of a given state would be.

It seems natural to regard states 'out there' as empirical entities, that is, as empirically distinguishable entities, where empirical distinctions are ones that make a possible difference to the world. (See [6, p. 58].) But then states are in general decomposable in several ways. It is even conceivable that no ultimate decomposition in simple parts exists.

In order to retain a one-to-one correspondence between states and complexes, suppose we were to give up Uniqueness. Now let us assume that the state of Willem-Alexander's loving Máxima has a single corresponding complex. Then this complex would not only be the completion of a binary relation, but of two unary relations as well. This, however, is not a desirable situation, since it obscures the interrelatedness of the complexes of a relation. ${ }^{1}$ This suggests that giving up Uniqueness should not be the first choice.

It may perhaps be argued that an identification of states with complexes should be taken in a more restricted sense, namely that it applies only to the class of atomic or elementary relations. It could be maintained that the states of such relations are mutually independent and that each state has a unique corresponding complex or is even identical to it. This view, however, is also problematic since it makes a strong metaphysical claim that is far from evident. No one has ever given a convincing example of an atomic relation. Furthermore, an account of other relations would still be needed. What Russell said about this issue in 'My Philosophical Development' is interesting:

I have come to think, however, that, although many things can be known to be complex, nothing can be known to be simple, and, moreover, that

\footnotetext{
${ }^{1}$ In Section 2.2, other examples will be given that illustrate this point more dramatically.
} 
statements in which complexes are named can be completely accurate, in spite of the fact that the complexes are not recognized as complex. [...] It follows that the whole question whether there are simples to be reached by analysis is unnecessary [24, p. 123].

Another attempt to 'save' a one-to-one correspondence between states and complexes, is to argue that each state 'out there' has a unique canonical corresponding complex. This could possibly be a complex with exactly one occurrence for each essentially involved object of the state. ${ }^{2}$ So, for the state of Narcissus's loving Narcissus we could get a canonical complex of the monadic self-love relation, and for Apollo's loving Daphne we could get a canonical complex of the dyadic love relation (unless Eros is essentially involved, as well). It may, however, not always be clear what the essentially involved objects of a state are. For example, if we accept the existence of disjunctive states, then the notion of essentially involved objects requires further clarification.

A related issue concerns the structure of thoughts. ${ }^{a}$ In 'Begriffsschrift' [11, Section 9], Frege discusses the notion of functions and considers the proposition that Cato killed Cato. Frege notes that if we think of "Cato" as replaceable at its first occurrence, then "to kill Cato" is the function, but we also have a function "to be killed by Cato" and a function "to kill oneself". There is, however, controversy about the question whether the different decompositions correspond to different thoughts.

José Luis Bermúdez [4, pp.94-95] claims that "“Cato killed Cato" can express four different thoughts", and that this also has to be Frege's position. On the other hand, Harold Hodes [14, p.162] argues that "Frege thought thoughts to be compositionally polymorphous". In the same vein, David Bell [3, p. 596] talks about "the mistake of taking function/argument analysis to reveal intrinsic structure", and admits that he is more and more convinced that thoughts do not have a determinate, intrinsic structure.

${ }^{a} \mathrm{Or}$ maybe it is the same issue. In 'Der Gedanke', Frege said: "Was ist eine Tatsache? Eine Tatsache ist ein Gedanke, der wahr ist."[12, pp.57-58] ("What is a fact? A fact is a thought that is true.")

\footnotetext{
${ }^{2}$ This approach may be advanced in particular by someone who regards (first-class) relations as universals, involving no particular particulars. (See [1, pp. 92-93].)
} 
By the way, Hodes [14, p. 176] leaves open the possibility that something is wrong with the notion of Fregean thought itself: "Perhaps the Fregean notion of a thought is a hybrid, born of confusions created by divided reference." With 'divided reference' he means, on the one hand, reference in terms of possible worlds, and, on the other hand, reference in terms of something like Carnap's notion of intensional isomorphism. I think the confusion pointed at might also be formulated as a confusion between the notions of relational states and relational complexes.

Wittgenstein struggled with a similar issue and talked about "Das alte Problem von Komplex und Tatsache" [28, entry 14.5.15]. Peter Simons [25, p.319] argues that "the Sachverhalte of the Tractatus are best seen not as atomic facts, but as atomic complexes". Furthermore, Simons [25, p. 335] claims that "Wittgenstein thought the Tractatus embodied a confusion between complex and fact". In the note 'Complex and fact' [27, pp. 301-303], Wittgenstein talks about a "muddle" and alludes to remarks in the Tractatus.

I started this short analysis of the correspondence between states and complexes with the assumption that states 'out there' are empirical entities. There might be good reasons to concede that many states 'out there' are nonempirical, but I think it is hard to deny that there is an important class of states 'out there' that are empirical. Therefore, my conclusion is that for a general account of relations we had better give up a one-to-one correspondence between states and complexes, and take another course.

In this paper, I present a rigorous theory of relations that explicitly distinguishes relational complexes from relational states. More specifically, $a$ relational complex is conceived as a structured perspective on a relational state. The theory is polymorphic in a strong sense, since it not only allows that a state 'out there' may belong to more than one relation, but also that for a given relation more than one relational complex can correspond to the same state.

I will not presuppose any particular view on the nature of relational states, or more generally, of states 'out there'. I will not even make any assumption about the existence of atomic complexes or states. The theory presented does not exclude the possibility that all states 'out there' are infinitely complex in the sense that all states are composed of simpler ones. Furthermore, it is not assumed (but not excluded either) that relational states themselves have an intrinsic structure. The theory can, for example, be combined with viewing states as David Lewis's sets of possible worlds [18], or as being akin to Kit Fine's worldly facts [6], or David Armstrong's states of affairs [1].

I call the theory the polymorphic theory of relations. It is presented in the form of a number of principles postulated throughout the paper (P-1 to P-14). In Appendix the principles are also listed for easy access. 


\section{Polymorphic View on Relations}

In the polymorphic view on relations, a key role is played by the notions relational states, relational complexes, and occurrences of objects. I will not try to give an exact definition of these notions. My objective is to characterize them in terms of a system of metaphysical principles.

\subsection{Structural Principles}

I postulate the following structural principles:

P-1 Each relation 'has' one or more relational complexes and each relational complex belongs to one and only one relation.

P-2 Each relational complex corresponds to one state 'out there' and each state 'out there' may correspond to one or more relational complexes.

P-3 Each relational complex may contain one or more occurrences of objects and each occurrence belongs to one and only one relational complex.

P-4 Each occurrence is the occurrence of one object and each object may be the content of one or more occurrences.

I call a state 'out there' that corresponds to a relational complex $\xi$ of a relation $\Re$ a relational state of $\Re$, and denote it as $S(\xi)$. I denote the occurrences of objects in a complex $\xi$ as $\operatorname{Oc}(\xi)$, and the object of an occurrence $\alpha$ as $\operatorname{Ob}(\alpha)$. Furthermore, I call the objects of the occurrences in a relational complex the objects of the complex, and the objects of the complexes of a relation the objects of the relation itself.

The number of occurrences in a relational complex $\xi$ is called the degree or adicity of $\xi$. The least upper bound of the degrees of the relational complexes of a relation $\Re$ is called the degree or adicity of $\Re$. If all relational complexes of a relation have the same degree, then the relation has a fixed degree, otherwise the relation has a variable degree. Relations of fixed degree are also called unigrade relations, and relations of variable degree are also called multigrade relations. An example of a multigrade relation is being surrounded by.

I consider a property as nothing but a monadic relation.

Remark 2.1 In order to keep the presentation simple, I ignore the possibility that each occurrence may be taken to have a type that corresponds to a domain of objects. The required changes here and in the principles further on are straightforward.

The structural principles are graphically represented in Fig. 1, which is a so-called entity-relationship diagram. ${ }^{3}$

\footnotetext{
${ }^{3}$ Entity-Relationship Modeling is an established technique to model the information needs of organizations. (I used it myself frequently as a consultant for Oracle Corporation.) More information about this technique can be found in [2] and [26].
} 
Fig. 1 Entity-relationship diagram for the polymorphic theory

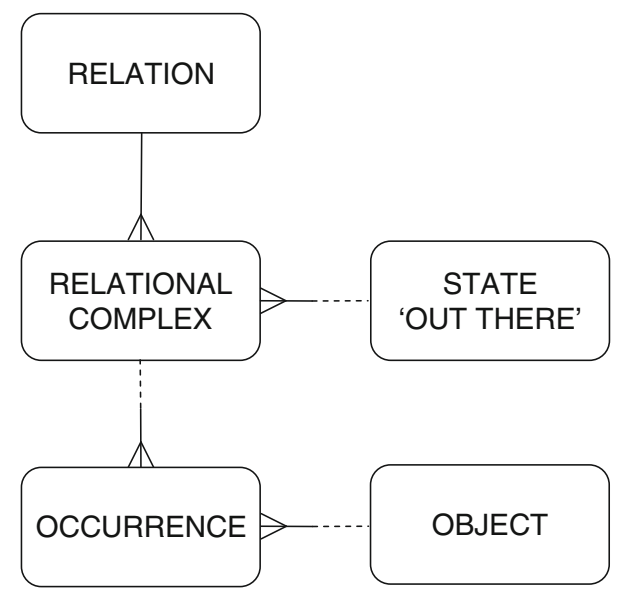

In such diagrams, a relationship between entities of type $\mathrm{A}$ and $\mathrm{B}$, where for each instance of $\mathrm{A}$ there is exactly one instance of $\mathrm{B}$, and for each instance of $\mathrm{B}$ there may be one or more instances of $\mathrm{A}$, is represented by:

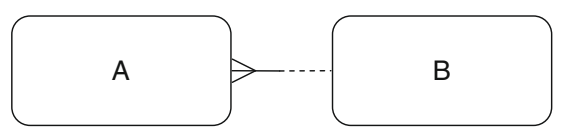

If for each instance of $B$ there is at least one related instance of $A$, then the right part of the line between the entities is also solid:

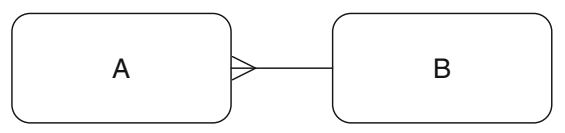

I don't expect that the structural principles will raise serious concerns, except perhaps Principle P-2. This principle will be evident for those who regard any set of possible worlds as a state 'out there', but those who think of states 'out there' as sparse, may perhaps consider it more adequate to make this correspondence optional. The exact consequences of this alternative choice are not immediately clear; we would have to find a satisfactory interpretation of complexes without corresponding states. Another possibility might be to let several states correspond to a given complex. For example, the complex of $x$ 's being red or round might correspond both to the state of $x$ 's being red and the state of $x$ 's being round.

The structural principles leave open whether a relation may have multiple relational complexes for the same state 'out there'. However, the principles in the next subsection will make this inevitable for certain relations. 


\subsection{Substitution Principles}

I do not assume that the occurrences in relational complexes are ordered, nor do I assume that relations have argument-places. The basic means by which relational complexes of a relation are thought to form a genuine unity is through substitution of objects for occurrences.

The notion of substitution is taken by Fine [7] as a primitive operation for his so-called antipositionalist view on relations. In models for relations I developed in $[15,17]$, substitution of objects also play a central role. There I assumed that substitution takes place directly in relational states, but here I take a different approach. The next principles concern substitution of objects for occurrences in relational complexes:

P-5 Any substitution of objects for occurrences in a relational complex results in exactly one relational complex of the same relation.

P-6 For any complex, the identity substitution results in the same complex.

P-7 Composition principle: If a substitution $\sigma$ in a relational complex $\xi$ results in $\xi^{\prime}$, then there is a bijection $\mu$ from the occurrences in $\xi$ to the occurrences in $\xi^{\prime}$, such that

(a) $\mu$ maps each occurrence $\alpha$ in $\xi$ to an occurrence of the object substituted by $\sigma$ for $\alpha$,

(b) any substitution $\sigma^{\prime}$ in $\xi^{\prime}$ gives the same result as substituting in $\xi$ for each occurrence $\alpha$ the object that $\sigma^{\prime}$ substitutes for $\mu(\alpha)$.

A mapping $\mu$ satisfying (a) and (b) of the composition principle is said to correspond to the substitution $\sigma$. Because of the injectivity of $\mu$, substitution is said to be coalescence-free.

Denotation Let $\Re$ be a relation, $\xi$ a relational complex of $\Re$, and $\sigma$ a mapping from the occurrences in $\xi$ to the objects of $\Re$. I denote the result of substituting in $\xi$ according to $\sigma$ as $\xi \cdot_{\Re} \sigma$ or $\xi \cdot \sigma$. Furthermore, I denote the composition of a mapping $f$ followed by a mapping $g$ as $f \cdot g$.

With this denotation, part (b) of Principle P-7 says that

$$
(\xi \cdot \sigma) \cdot \sigma^{\prime}=\xi \cdot\left(\mu \cdot \sigma^{\prime}\right) .
$$

Let me make a few comments on the substitution principles.

Ad P-5. Certain substitutions result in complexes corresponding to impossible states. This happens, for example, when substituting $a$ for the occurrence of $b$ in the complex $a$ 's being next to $b$. If you want to exclude impossible states, then you could of course postulate a weaker principle than P-5, namely one stating that any substitution results in at most one relational complex.

Instead of assuming that any occurrence is an occurrence for which objects can be substituted, we might also consider introducing 'fixed' or 'hidden' occurrences of objects. In Section 3.2 the operation of hiding occurrences will be discussed. 
Ad P-7. The composition principle forms the heart of the theory developed in this paper. It expresses the way different relational complexes form some kind of unity or resemble each other. This may be illustrated as follows:

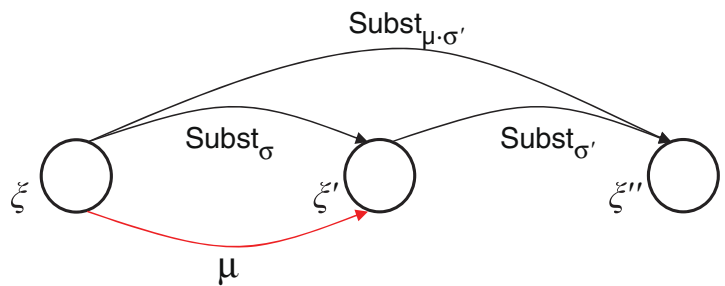

It should be noticed that for a given substitution $\sigma$, a corresponding mapping $\mu$ is not necessarily unique; for symmetric relations the uniqueness may fail.

An important consequence of the principles P-1 to P-7 is that - for an empirical account of states 'out there'-certain relations are inevitably polymorphic in the sense that some states need to have more than one corresponding complex within the same relation:

Example 2.2 Suppose that states 'out there' are sets of possible worlds. Let $\Re$ be the parthood relation. The state that my nose is a part of myself has a corresponding complex with one occurrence of my nose and one of myself. It seems reasonable to assume that in no possible world I am a part of my nose and neither are you. But this means that the relation $\Re$ has a state-to wit, the empty state-that necessarily has more than one corresponding complex within $\Re$.

It could be objected that in the example we are dealing with a borderline case, since the state with multiple complexes is a vacuous state. So, let me also give another example.

Example 2.3 Let $\Re$ be the binary love relation with states $x \rightarrow$ $\gg$, and let $\Re^{\prime}$ be the unary relation with states $x \rightarrow d$ with $d$ a fixed object. Now consider the conjunction of $\Re$ and $\Re^{\prime}{ }^{4}$ Let $a, c$ be distinct objects. Then the conjunctive state $a \gg d \otimes c \gg d$ has in this relation two corresponding complexes.

To see this, let $b$ be another object and let $\xi$ be a complex corresponding to the state $a \gg b \otimes c \gg d$. This complex has three occurrences. We might say that the occurrence of $c$ is connected to a hidden occurrence of $d$. If we substitute in $\xi$ the object $d$ for the occurrence of $b$, then in the resulting complex the occurrence of $c$ is still connected to a hidden occurrence of $d$. But if we would have started with a complex for the state $c \gg b \otimes a \gg \rightarrow d$,

\footnotetext{
${ }^{4}$ In Section 3, conjunction of relations and hiding occurrences of objects will be discussed in more detail.
} 
then substituting $d$ for $b$, would have given a complex with the occurrence of $a$ connected to a hidden occurrence of $d$. Thus, this complex is not identical to $\xi$, but they obviously have the same corresponding state 'out there'.

One could adopt an extremely refined notion of states 'out there' and argue that in the example the relations $\Re$ and $\Re^{\prime}$ have no states in common. But if one regards states 'out there' as really being 'out there', this view is highly implausible. Alternatively, one could disallow 'object-involving' relations like $\Re^{\prime}$, but I see no reason to do so for a general theory of relations.

In Principle P-7 the mapping $\mu$ is required to be bijective. As a result, complexes that can be obtained one from the other by substitution have a very uniform structure. I consider this as an advantage, although this may also give rise to multiple complexes for the same state. For example, consider a ternary relation $\Re$ with all states being (commutative) conjunctions of the form $x \gg y \oplus y \gg z$. Then for distinct objects $a, b$ the state $a \gg b \oplus b \gg a$ has two corresponding complexes in $\Re$, namely a complex with one occurrence of $a$ and two of $b$, and another complex with two occurrences of $a$ and one of $b$.

In [17] I questioned the validity of a coalescence-free account. My expectation was that by allowing coalescence we could develop a satisfactory theory without multiple complexes for the same state within a single relation. But, as we have seen in Examples 2.2 and 2.3, then not all relational states can be conceived of as empirical entities. Of course, there may be other arguments for allowing coalescence for a general theory of relations. One of them could be the way substitution works for set-like relations. I discuss this issue in the next subsection.

\subsubsection{Understanding Substitution}

The notion of substitution may need further explanation. Postulating a notion as primitive does not imply that its meaning is immediately clear. As I said before, I regard a relational complex as a structured perspective on a state 'out there'. But what does this mean? It might be tempting to entertain a picturelike representation for a relational complex, and to consider substitution simply as literally replacing in such a picture objects by other objects. But how accurate is such a representation?

Consider the adjacency relation. Suppose you have in front of you a cup adjacent to a glass. Then switching these objects obviously changes the scene. But, if the objects of a corresponding relational complex are switched, then it seems reasonable to assume that the result should be exactly the same relational complex. However, in working with a naive picture-like representation this may not be so easy to accomplish. Or suppose you want to substitute a chair for the glass. Then in such a representation you may have trouble deciding where exactly to put the chair. A naive picture-like representation may also be problematic for other relations. Take, for example, the relation of an object $x$ being wedged between objects $y$ and $z$. Then substituting for $x$ 
an object of a different size, may require that the other objects are moved in the representation as well. What these examples show is that a picturelike representation for relational complexes may only be adequate if it is sufficiently abstract to accommodate these situations.

Another issue is how to understand substitution for a relation like forming a group. Suppose the group consisting of Groucho, Harpo and Chico has a corresponding complex with one occurrence for each person. Now what do we get if we substitute Groucho for the occurrence of Harpo? According to the substitution principles we get a complex with two occurrences of Groucho and one of Chico. But the state of Groucho and Chico forming a group also has a corresponding complex with one occurrence of each. Apparently the states with a set-like character have corresponding complexes with a multiset-like character. This might look a bit odd or artificial, but I don't see this as an objection to a coalescence-free account of substitution. Rather, I would say that each complex reflects precisely the way it can be obtained by substitution. In addition, we can of course assign to each state a canonical complex with one occurrence for each person. Alternatively, we could consider leaving substitution undefined in this case and postulate that substitution is only a partial function. (See also the discussion above of P-5.) In the next subsection, a complementary operation will be discussed that is relevant for relations like forming a group, namely subtracting occurrences from complexes.

\subsection{Connectivity Principles}

A relation is given as a unity. But what exactly constitutes this unity; how do the complexes of a relation form a coherent whole?

For most ordinary relations, the relational complexes all have the same finite number of occurrences of objects. For this class of relations, the next principle characterizes the interrelatedness between the relational complexes.

P-8 For any relation $\mathfrak{R}$ of fixed finite degree, any relational complex of $\mathfrak{R}$ can be obtained from any other relational complex of $\Re$ via substitution.

With this principle it becomes possible to give positional representations for any relation of fixed finite degree. (See [15] and [16].)

The principle is limited to relations of finite degree because certain relations of fixed infinite degree may have for a subset of its occurrences a kind of variable degree. Take for example the conjunction of a multigrade relation whose complexes have finite degree and a relation of fixed infinite degree. For such a conjunctive relation a connectivity principle like P-8 obviously does not hold. Another, perhaps more natural, counterexample is the relation of $x_{1}, x_{2}, \ldots, x_{n}$ being a finite segment of $y_{1}, y_{2}, \ldots$, with $n$ variable and $y_{1}, y_{2}, \ldots$ an infinite list.

Note that by Principle P-8, a structure with complexes of the form $x_{1}, \ldots, x_{n}$ playing against $y_{1}, \ldots, y_{m}$ with $n, m$ variable, but $n+m$ fixed, is not a relation. However, I do not consider this limitation of particular metaphysical significance. The polymorphic theory presented in this paper could be relaxed 
so that structures like this would be relations as well. A possibility could be to accept as a relation any substructure of a relation as long as it is closed under substitution.

For an arbitrary relation, I do not have a satisfactory solution for characterizing the unity of its complexes. However, for multigrade relations with a set-like character, like forming a team, collectively supporting, and being surrounded by the notion of subtracting occurrences may be helpful. We simply remove certain occurrences from a relational complex and get another complex.

For the operation of subtraction of occurrences additional principles can be proposed, in particular, a generalized composition principle that involves substitutions and/or subtractions. Care should be taken, however, not to confuse the subtraction of an occurrence with substituting the object of the occurrence with 'nothing', resulting in some kind of 'empty' occurrence.

For the multigrade relation of $x_{1}, \ldots, x_{n}$ forming a team, there is for any pair of relational complexes a substitution and/or subtraction from one of the complexes to the other. But for some other multigrade relations, we may need a third relational complex to make a connection. This might even be the case when the first two relational complexes have the same finite degree. We have such a situation for the relation of $x_{1}, \ldots, x_{n}$ playing against $y_{1}, \ldots, y_{m}$ with $n, m$ variable.

As a connectivity principle for multigrade relations with a (multi)set-like character, it could be proposed that the relational complexes (with substitutions and/or subtractions as arrows) form a weakly connected graph. ${ }^{5}$

An important question is whether subtraction is a basic operation for multigrade relations. Kit Fine remarked [private communication, November 2008] that he is inclined to regard the possibility of subtraction as a symptom of variable adicity and not as an explanation. I agree that subtraction is maybe not an explanation of variable adicity, but it may be a way to characterize the unity of the relational complexes of a multigrade relation.

Alternatively, could we not simply deny that there are multigrade relations? Could we not say that a relation like being surrounded by has only two occurrences: one for the surrounded entity and one for the set of surrounders?

By taking sets, wholes or aggregates as compound objects of relations, relations that at first sight appear to have variable adicity may turn out to have in fact a fixed adicity. The strategy, which goes back to Bolzano [5, pp. 2-3] and to Frege [10, p. 246], looks attractive, but does it work?

A difficulty with the strategy has been brought forward by Fraser MacBride [20, pp.583-584]. He points out that rendering a multigrade relation unigrade appears to presuppose the existence of at least one apparently multigrade relation, namely the constitution relation, to manufacture compound objects. To remove the multigrade appearance of the constitution relation would involve another multigrade relation, and so on. In other words, a complete removal of

\footnotetext{
${ }^{5} \mathrm{~A}$ directed graph is called weakly connected if its underlying undirected graph is connected.
} 
multigrade relations leads to an infinite regress. But, as Macbride notes, the regress can be avoided by admitting a multigrade relation of constitution.

There may, however, be an additional complication with rendering multigrade relations to unigrade relations if we start with a multigrade relation where the $n$ objects have a relative order, but no object can in any genuine sense be said to be the first one. This is, for example, the case for a 'linked' love relation with states $x_{1} \gg x_{2} \gg \ldots . \gg x_{n}$. If we want to render this relation as a unigrade relation, then the question is whether we have a neutral choice for the structure of the compound objects. Choosing sequences seems a bad idea, because then the compound objects of the resulting relation would have a first element, and this would conflict with taking the love relation as directionless. ${ }^{6}$ Unfortunately, I do not see an acceptable artifact-free alternative. We could consider to introduce a new sort of compound object with the required structure, but it is not very clear to me what the ontology of such compound objects would be.

For characterizing arbitrary multigrade relations, the notion of subtracting occurrences is too limited. But, as I said, I have as yet no satisfactory general solution.

\subsection{Identity Criteria}

Because more than one relational complex for a given relation may correspond to the same relational state, identity criteria for relations and relational complexes may be useful to prevent an unjustifiable abundance of relations with exactly the same relational states. The basic idea behind the criteria proposed is that a relational complex is a purely extensional notion.

\subsubsection{Identity of Relations}

Relations with fully matching complexes-with respect to substitution-are called qualitatively the same. They are formally defined as follows.

Definition 2.4 Relations $\Re, \Re^{\prime}$ are called qualitatively the same (or qualitatively identical) if there is a bijection $\pi$ from the complexes of $\Re$ to the complexes of $\Re^{\prime}$ such that for every complex $\xi$ of $\Re^{7}$

1. $\mathrm{S}(\pi(\xi))=\mathrm{S}(\xi)$,

2. there is a bijection $\tau: \mathrm{Oc}(\xi) \rightarrow \mathrm{Oc}(\pi(\xi))$ such that

(a) for each occurrence $\alpha$ in $\xi, \mathrm{Ob}(\tau(\alpha))=\mathrm{Ob}(\alpha)$,

(b) for each substitution $\sigma$ in $\xi, \pi(\xi \cdot \sigma)=\pi(\xi) \cdot\left(\tau^{-1} \cdot \sigma\right)$.

\footnotetext{
${ }^{6}$ This argument is related to Fine's objection against what he calls the standard view on relations [7].

${ }^{7}$ Recall that $\mathrm{S}(\xi)$ denotes the state corresponding to the complex $\xi$, that $\mathrm{Oc}(\xi)$ denotes the occurrences of objects in $\xi$, and that $\mathrm{Ob}(\alpha)$ denotes the object of the occurrence $\alpha$.
} 
The love relation and the hate relation are obviously not qualitatively the same, because they have different states. But let $\Re$ be a unary relation with $\Re x$ being the complex $x$ 's loves himself or $x$ does not love himself, and let $\Re^{\prime}$ be a unary relation with $\Re^{\prime} x$ being the complex $x$ 's hates himself or $x$ does not hate himself, and suppose that for any $x$ the states corresponding to the complexes $\Re x$ and $\Re^{\prime} x$ are the same. Then $\Re$ and $\Re^{\prime}$ are qualitatively the same.

The next lemma shows that there is a completely structure-preserving correspondence between the occurrences of objects in matching complexes of qualitatively the same relations.

Lemma 2.5 Suppose $\mathfrak{R}$ and $\mathfrak{R}^{\prime}$ are qualitatively the same. Then in the following commuting diagram

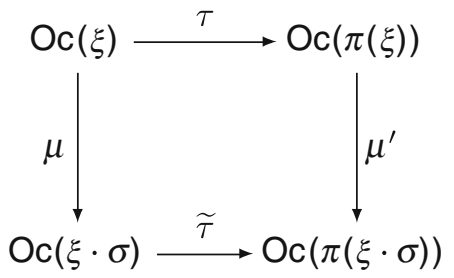

with $\pi, \tau, \tilde{\tau}$ in line with Definition 2.4, with $\mu$ a mapping corresponding to substitution $\sigma$ in $\xi$, and with $\mu^{\prime}=\tau^{-1} \cdot \mu \cdot \tilde{\tau}$, the mapping $\mu^{\prime}$ corresponds to substitution $\tau^{-1} \cdot \sigma$ in $\pi(\xi)$.

Proof Because $\mu^{\prime}$ maps each occurrence $\alpha$ in $\pi(\xi)$ to an occurrence of the object $\left(\tau^{-1} \cdot \sigma\right)(\alpha)$, Condition (a) of the composition principle P-7 holds for $\mu^{\prime}$. To prove Condition (b) of P-7, let $\sigma^{\prime}$ be an arbitrary substitution in $\pi(\xi \cdot \sigma)$. Then

$$
\begin{aligned}
\left(\pi(\xi) \cdot\left(\tau^{-1} \cdot \sigma\right)\right) \cdot \sigma^{\prime} & =\pi(\xi \cdot \sigma) \cdot \sigma^{\prime} \\
& =\pi\left((\xi \cdot \sigma) \cdot\left(\tilde{\tau} \cdot \sigma^{\prime}\right)\right) \\
& =\pi\left(\xi \cdot\left(\mu \cdot \tilde{\tau} \cdot \sigma^{\prime}\right)\right) \\
& =\pi(\xi) \cdot\left(\mu^{\prime} \cdot \sigma^{\prime}\right) .
\end{aligned}
$$

Thus, because $\mu^{\prime}$ is bijective, $\mu^{\prime}$ corresponds to substitution $\tau^{-1} \cdot \sigma$ in $\pi(\xi)$.

I postulate the following identity criterion for relations:

P-9 Relations are identical if and only if they are qualitatively the same.

The criterion expresses that a relation is completely determined by the network of interconnections of substitution in which the complexes with their corresponding states stand to each other. We might say that the complexes themselves have no internal complexity.

A bolder identity criterion could be considered, namely that relations are identical if and only if they have the same states. This could not be right, however, for some accounts of states 'out there'. For suppose that states 'out 
there' are sets of possible worlds. Then the relation $\Re$ with complexes $x$ and $y$ are the same number and the relation $\Re^{\prime}$ with complexes $x$ and $y$ are not the same number have the same states, namely the set of all possible worlds and the empty set. But $\mathfrak{R}$ and $\Re^{\prime}$ are obviously not identical.

The identity criterion P-9 is stated for arbitrary relations. To play on the safe side, we might consider to limit it to relations where all complexes are connected to each other via substitution. I consider this as an issue for future investigation.

\subsubsection{Identity of Relational Complexes}

For relational complexes I also define the notion of qualitative sameness. The idea is that two complexes of the same relation are qualitatively the same if substitutions in them are fully matching.

Definition 2.6 Relational complexes $\xi$ of $\Re$ and $\xi^{\prime}$ of $\Re^{\prime}$ are called qualitatively the same (or qualitatively identical) if there is a bijection $\tau$ from the occurrences in $\xi$ to the occurrences in $\xi^{\prime}$ such that

1. for each occurrence $\alpha$ in $\xi, \mathrm{Ob}(\tau(\alpha))=\mathrm{Ob}(\alpha)$,

2. a substitution $\sigma$ in $\xi$ is defined iff the $\tau$-corresponding substitution in $\xi^{\prime}$ is defined, and $\mathrm{S}(\xi \cdot \sigma)=\mathrm{S}\left(\xi^{\prime} \cdot\left(\tau^{-1} \cdot \sigma\right)\right)$.

A non-trivial (but somewhat artificial) example of complexes that are qualitatively the same can be obtained as follows. Consider the ternary relation $\Re$ with $\Re x y z$ the complex $x$ is not identical to itself and $y$ loves $z$. Then in a possible worlds conception of states 'out there', the complexes $\Re x y z$ and $\Re x z y$ have the same corresponding state. It follows that the complexes are qualitatively the same.

Note that qualitative sameness of relational complexes is an equivalence relation. Moreover, the equivalence is preserved under substitution:

Lemma 2.7 Suppose $\xi$ and $\xi^{\prime}$ are qualitatively the same relational complexes, and let $\tau$ be as in Definition 2.6. Then for every substitution $\sigma$ in $\xi$, the complexes $\xi \cdot \sigma$ and $\xi^{\prime} \cdot\left(\tau^{-1} \cdot \sigma\right)$ are qualitatively the same as well.

Proof Let $\mu: \mathrm{Oc}(\xi) \rightarrow \mathrm{Oc}(\xi \cdot \sigma)$ correspond to substitution $\sigma$ in $\xi$, and let $\mu^{\prime}$ correspond to substitution $\tau^{-1} \cdot \sigma$ in $\xi^{\prime}$. Then, by Principle P-7, the mapping $\tilde{\tau}=\mu^{-1} \cdot \tau \cdot \mu^{\prime}$ is a bijection. So, we have the following commuting diagram:

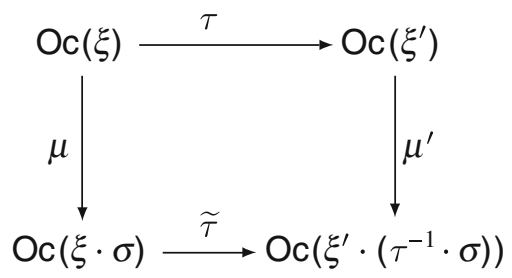


The mapping $\tilde{\tau}$ obviously fulfills Condition 1 of Definition 2.6. Furthermore,

$$
\begin{aligned}
\mathrm{S}\left((\xi \cdot \sigma) \cdot \sigma^{\prime}\right) & =\mathrm{S}\left(\xi \cdot\left(\mu \cdot \sigma^{\prime}\right)\right) \\
& =\mathrm{S}\left(\xi^{\prime} \cdot\left(\tau^{-1} \cdot \mu \cdot \sigma^{\prime}\right)\right) \\
& =\mathrm{S}\left(\xi^{\prime} \cdot\left(\mu^{\prime} \cdot \tilde{\tau}^{-1} \cdot \sigma^{\prime}\right)\right) \\
& =\mathrm{S}\left(\xi^{\prime} \cdot\left(\tau^{-1} \cdot \sigma\right) \cdot\left(\tilde{\tau}^{-1} \cdot \sigma^{\prime}\right)\right) .
\end{aligned}
$$

Thus, $\widetilde{\tau}$ also fulfills Condition 2 of Definition 2.6.

For many relations, we neither want nor need multiple relational complexes for the same state. For example, for the adjacency relation we want that the state of $a$ 's being next to $b$ has only one relational complex. Substituting $a$ for the occurrence of $b$ and $b$ for the occurrence of $a$ should give the same relational complex. To take care of such situations, I propose the following identity criterion for relational complexes:

P-10 Relational complexes that belong to the same relation are identical if and only if they are qualitatively the same.

Principle $\mathrm{P}-10$ has the following nice property. If $\Re$ were to be a relation that does not fulfill P-10, then $\Re$ can be stripped down to a structure $\breve{\Re}$ that fulfills this principle, and in which everything that seems essential to $\Re$ is preserved. In particular, $\breve{\Re}$ also fulfills the composition principle P-7.

To see this, define $\breve{\mathfrak{R}}$ as a relation-like structure whose complexes are arbitrarily chosen representatives of qualitatively the same complexes in $\Re$, and with substitution defined by

$$
\xi \cdot_{\mathfrak{\Re}^{\prime}} \sigma=\xi^{\prime} \text { with } \xi^{\prime} \text { qualitatively the same as } \xi \cdot \Re \sigma \text {. }
$$

For $\xi \cdot_{\Re} \sigma$ and $\xi \cdot_{\mathfrak{\Re}} \sigma$ let $\tau$ be a bijection as in Definition 2.6. Let $\mu$ be a mapping that corresponds-in accordance with Principle P-7-to the substitution $\sigma$ applied to $\xi$. Then $\left(\xi \cdot_{\breve{\Re}} \sigma\right) \cdot_{\breve{\Re}} \sigma^{\prime}=\xi \cdot_{\mathfrak{\Re}}\left(\mu \cdot \tau \cdot \sigma^{\prime}\right)$. It follows that $\breve{\Re}$ also fulfills the composition principle.

Note that to obtain a relation as a result of this stripping process, it is essential that the stripping is done simultaneously for all relational complexes of $\Re$.

The next lemma shows that the identity of a relation can be expressed in terms of qualitatively the same complexes.

Lemma 2.8 Let $\Re$ and $\Re^{\prime}$ be relations. If for every complex in $\Re$ there is a qualitatively identical complex in $\Re^{\prime}$, and vice versa, then $\Re$ and $\Re^{\prime}$ are identical.

Proof Assume that for every complex $\xi$ in $\Re$ there is a qualitatively identical complex $\xi^{\prime}$ in $\Re^{\prime}$, and vice versa. Then, since qualitative sameness of complexes is an equivalence relation, it follows from Principle P-10 that $\xi^{\prime}$ is the only complex in $\mathfrak{R}^{\prime}$ that is qualitatively the same as $\xi$. Because, conversely, $\xi$ is also the only complex in $\Re$ that is qualitatively the same as $\xi^{\prime}$, there is a bijection 
$\pi$ that maps the complexes of $\Re$ to their qualitatively identical counterparts in $\Re^{\prime}$. Now, by using Lemma 2.7 , we see that this bijection $\pi$ makes $\Re$ and $\mathfrak{R}^{\prime}$ qualitatively the same relations. Thus, by P-9, they are identical.

It is interesting to note that, as a consequence of Principle P-10, the identity mapping is the only bijection $\pi$ in Definition 2.4 that makes a relation qualitatively identical to itself.

\subsubsection{Justifying the Identity Criteria}

The identity criteria for relations and for relational complexes have some consequences that at first sight may strike us as odd:

Example 2.9 Let $\Re_{0}$ be a nullary relation with as its only state the state of everyone's loving and trusting everyone. If the conjunction of this relation with the binary love relation is again a relation, say $\Re_{1}$, then how does $\Re_{1}$ look like? It seems reasonable to assume that $\Re_{1}$ is a binary relation. Furthermore, if relational states are conceived of as sets of possible worlds, then $\Re_{1}$ would obviously also have just one state. Now by the identity criterion for relational complexes, it would also have for any (unordered) pair of objects $a, b$ just one complex with one occurrence of $a$ and one $b$ (and two of $a$ if $a$ and $b$ are identical). So, if $\alpha, \beta$ are the occurrences in a complex in $\Re_{1}$, then we cannot say which of them fulfills the role of lover and which the role of beloved.

In a similar way, we can define a relation $\Re_{2}$ as the conjunction of $\Re_{0}$ with the binary trust relation. According to the identity criterion for relations, $\Re_{1}$ and $\Re_{2}$ would be identical. Thus, in the resulting relation no traces are left of either a 'love' or a 'trust' origin.

One might wonder whether a more refined account of relations that retains more of the origin of the underlying complexes and occurrences would be more adequate. Although I do not exclude the possibility of such an account, I think there are strong arguments in favor of the proposed identity criteria.

An important feature of the proposed criteria is their definiteness: the question whether two relations or relational complexes are identical has a clear, definite answer. Another strong feature of the proposed criteria is, as we will see in Section 3, that they allow us to define various operations on relations in a very natural way in terms of operations on relational states.

\section{Operations on Relations}

I discuss operations on relations that I consider as very basic, namely conjunction, disjunction, negation, and restriction. With these operations all kinds of complex relations can be constructed. Of course, other basic operations are conceivable as well. Besides, it is not excluded that certain relations supervene on others without any possible reduction. By this I mean that the existence 
of certain relations might be entailed by other relations, but that they are not explicitly definable in terms of these other relations.

\subsection{Logical Operations}

Suppose we want to define the conjunction of relations with sets of complexes $C_{1}$ and $C_{2}$. Then for the complexes of the conjunction we cannot simply take $C_{1} \times C_{2}$, because this may yield too many complexes, and because the ordering in the elements is undesirable. For the occurrences in the conjunction of complexes $\xi$ and $\xi^{\prime}$ we also cannot simply take the union of the occurrences in $\xi$ and $\xi^{\prime}$, because $\xi$ and $\xi^{\prime}$ may be identical, and because other complexes may have the same conjunction. We must be more cautious, but we can define logical operations on relations in a clear and uniform way in terms of logical operations on states:

Definition 3.1 Let $\Re_{1}, \ldots, \Re_{n}$ be relations with sets of states $S_{1}, \ldots, S_{n}$ and sets of complexes $C_{1}, \ldots, C_{n}$. Let $f$ be a mapping from $S_{1} \times \ldots \times S_{n}$ to some set of states $S$.

We say that a relation $\Re$ with set of complexes $C$ is induced by $f$ if there is a surjection $\rho: C_{1} \times \ldots \times C_{n} \rightarrow C$, and for every $\xi_{1} \in C_{1}, \ldots, \xi_{n} \in C_{n}$, a bijection $\tau: X \rightarrow \operatorname{Oc}\left(\rho\left(\xi_{1}, \ldots, \xi_{n}\right)\right)$, with $X=\bigcup_{i=1}^{n}\left(\mathrm{Oc}\left(\xi_{i}\right) \times\{i\}\right)$, the disjoint union of $\mathrm{Oc}\left(\xi_{1}\right), \ldots, \operatorname{Oc}\left(\xi_{n}\right)$, such that

1. for each $(\alpha, i) \in X, \mathrm{Ob}(\tau(\alpha, i))=\mathrm{Ob}(\alpha)$,

2. for each substitution $\sigma$ in $\rho\left(\xi_{1}, \ldots, \xi_{n}\right)$ and $\tau$-corresponding substitutions, $\mathrm{S}\left(\rho\left(\xi_{1}, \ldots, \xi_{n}\right) \cdot \sigma\right)=f\left(\mathrm{~S}\left(\xi_{1} \cdot \sigma_{1}\right), \ldots, \mathrm{S}\left(\xi_{n} \cdot \sigma_{n}\right)\right)$.

The identity criteria for relations and relational complexes guarantee that $\Re$ is thus uniquely defined:

Lemma 3.2 For the given collection of relations $\Re_{1}, \ldots, \Re_{n}$, there is at most one relation $\Re$ induced by $f$.

Proof Suppose that $\Re$ and $\mathfrak{R}^{\prime}$ are both induced by $f$. Then for every complex $\rho\left(\xi_{1}, \ldots, \xi_{n}\right)$ in $\Re$ there is a qualitatively identical complex $\rho^{\prime}\left(\xi_{1}, \ldots, \xi_{n}\right)$ in $\Re^{\prime}$, and vice versa. So, by Lemma $2.8, \mathfrak{R}$ and $\mathfrak{R}^{\prime}$ are identical.

If for given relations $\Re_{1}, \ldots, \Re_{n}$ there is a relation induced by $f$, then it is denoted as $f\left(\Re_{1}, \ldots \Re_{n}\right)$ and its complexes are denoted as $f\left(\xi_{1}, \ldots \xi_{n}\right)$.

Note that the degree of $f\left(\Re_{1}, \ldots \Re_{n}\right)$ is equal to the sum of the degrees of the relations $\Re_{1}, \ldots \Re_{n}$. Also note that if $f$ is the identity mapping from $S_{1}$ to $S_{1}$, then $f\left(\Re_{1}\right)=\Re_{1}$.

Suppose $f$ is a binary mapping with for all states $f\left(s_{1}, s_{2}\right)=f\left(s_{2}, s_{1}\right)$. Then clearly also for all complexes $f\left(\xi_{1}, \xi_{2}\right)=f\left(\xi_{2}, \xi_{1}\right)$, if defined. The next lemma establishes a more general similarity between identities of compositions of states and identities of compositions of complexes. 
Lemma 3.3 Let $\Re_{1}, \ldots, \Re_{n}$ be relations with sets of states $S_{1}, \ldots, S_{n}$ and sets of complexes $C_{1}, \ldots, C_{n}$. Let $\pi$ be a permutation of $\{1, \ldots, n\}$, and let $f_{1}, \ldots, f_{m}, g, h$ be mappings such that for all $s_{1} \in S_{1}, \ldots, s_{n} \in S_{n}$,

$$
g\left(f_{1}\left(s_{\pi(1)}, \ldots, s_{\pi(k)}\right), f_{2}\left(s_{\pi(k+1)}, \ldots\right), \ldots, f_{m}\left(\ldots, s_{\pi(n)}\right)\right)=h\left(s_{1}, \ldots, s_{n}\right) .
$$

Then for all $\xi_{1} \in C_{1}, \ldots, \xi_{n} \in C_{n}$,

$$
g\left(f_{1}\left(\xi_{\pi(1)}, \ldots, \xi_{\pi(k)}\right), f_{2}\left(\xi_{\pi(k+1)}, \ldots\right), \ldots, f_{m}\left(\ldots, \xi_{\pi(n)}\right)\right)=h\left(\xi_{1}, \ldots, \xi_{n}\right),
$$

if defined.

Proof Suppose $g\left(f_{1}\left(\Re_{\pi(1)}, \ldots\right), \ldots, f_{m}\left(\ldots, \Re_{\pi(n)}\right)\right)$ and $h\left(\Re_{1}, \ldots, \Re_{n}\right)$ are relations. Let us call them $\mathfrak{R}$ and $\mathfrak{R}^{\prime}$, respectively. Let $\rho$ be the mapping from the complexes of $\Re$ to the complexes of $\Re^{\prime}$ defined by

$$
g\left(f_{1}\left(\xi_{\pi(1)}, \ldots,\right), \ldots, f_{m}\left(\ldots, \xi_{\pi(n)}\right)\right) \mapsto h\left(\xi_{1}, \ldots, \xi_{n}\right)
$$

From the given equations for the states, it follows that $\rho$ is well-defined. Now let $\sigma$ be an arbitrary substitution in a complex $\xi$ of $\Re$, and let $\sigma^{\prime}$ be the corresponding substitution in $\rho(\xi)$. It is easy to see that $\mathrm{S}(\xi \cdot \sigma)=\mathrm{S}\left(\rho(\xi) \cdot \sigma^{\prime}\right)$. It follows that $\Re$ and $\Re^{\prime}$ are qualitatively the same relations, and thus by P-9 they are identical. The equations for the complexes follow directly from this.

In the next subsections the lemma will be applied to standard logical operations, but let me already mention a few applications: (1) If the conjunction of any two states is commutative, then the conjunction of complexes is commutative as well. (2) If the double negation of any state is the state itself, then the double negation of any complex is the complex itself. (3) If we have De Morgan's laws for all states, like $-\left(s \otimes s^{\prime}\right)=-s \otimes-s^{\prime}$, then we also have identities like $-\left(\xi \otimes \xi^{\prime}\right)=-\xi \otimes-\xi^{\prime}$. But note that if for all $s, s \otimes s=s$, then this does not imply that $\xi \otimes \xi=\xi$.

\subsubsection{Conjunction of Relations}

Russell [21, pp.209-211] sees no reason to accept conjunctive facts, for the truth of a proposition $p \otimes q$ depends only on the fact corresponding to $p$ and the fact corresponding to $q$. Wittgenstein is also said to consider conjunctive facts as unnecessary (for example in [19, p.382]), but he does not deny their existence, since he writes in response to a question of Russell [28, p.130]: "Sachverhalt is, what corresponds to an Elementarsatz if it is true. Tatsache is 
what corresponds to the logical product of elementary props when this product is true." A different opinion is held by Armstrong. He accepts conjunctive facts and points out that there could perhaps be conjunctive universals 'all the way down' [1, p. 32].

For the theory developed in this paper, I assume that for all states 'out there' $s, s^{\prime}$ the conjunction $s \otimes s^{\prime}$ is a well-defined state 'out there', although certain conjunctive states might be more natural than others.

Which properties you attribute to the conjunctive states 'out there' will depend on your specific view, but commutativity and associativity seem to be evident. Frege, for example, remarks about commutativity for compound thoughts [13, p. 89]: "Dass "B und A" denselben Sinn hat wie "A und B" sieht man ein ohne Beweis nur dadurch, dass man sich des Sinnes bewusst wird.", and he adds in a footnote [note 5]: "Ein anderen Fall dieser Art ist der, dass "A und A" denselben Sinn hat wie "A"."

I define the conjunction of relations in terms of the conjunction of their states, and by making use of Definition 3.1:

Definition 3.4 Let $\Re$ and $\Re^{\prime}$ be relations. Then, if it exists, the conjunction of $\Re$ and $\Re^{\prime}$ is defined as the relation induced by the conjunctive operation on states. It is denoted as $\Re \otimes \Re^{\prime}$, and its complexes as $\xi \otimes \xi^{\prime}$.

I postulate that the conjunction of any pair of relations is also a relation:

\section{P-11 Relation $\Re \otimes \Re^{\prime}$ exists for any relations $\mathfrak{R}, \Re^{\prime}$.}

As we have seen in Example 2.3, if for each relation each relational state may only have one corresponding relational complex, then the conjunction of relations would not always exist. Also the next example shows that a conjunction of relations might introduce multiple relational complexes of the same relational state.

Example 3.5 Consider the conjunctive relation $\Re \otimes(\Re \oplus \Re)$. Suppose conjunction of states is commutative, associative and idempotent. Then the complexes $\xi \otimes\left(\xi \otimes \xi^{\prime}\right)$ and $\xi^{\prime} \otimes\left(\xi \otimes \xi^{\prime}\right)$ correspond to the same relational state, but, in many cases, the complexes will be distinct. Note that if in a relation no multiple relational complexes were allowed for a single relational state, then substitution would in these cases inevitable lead to a coalescence of occurrences.

\footnotetext{
${ }^{8}$ Translation [9, pp. 4-5]: "That "B and A" has the same sense as "A and B" can be seen without proof by merely taking account of the sense." Note 5: "Another case of this sort is that "A and A" has the same sense as "A"."
} 
The next theorem follows directly from Lemma 3.3.

Theorem 3.6 If conjunction of states is commutative (associative), then conjunction of complexes is commutative (associative) as well.

So, it also follows that if conjunction of states is commutative (associative), then conjunction of relations is commutative (associative) as well.

Note that the theorem is not true for every theory of relations. For a theory where the arguments of the relations come in a certain order, conjunction of complexes and of relations is obviously not commutative. Also for a theory where the relations come with argument-places, conjunction of complexes is not commutative, but conjunction of relations can be commutative, provided that there are suitable identity criteria for sets of argument-places.

Besides a conjunction of two relations, we also might define a conjunction of an infinite number of relations, provided that we accept an infinite conjunction of states 'out there'.

\subsubsection{Disjunction of Relations}

Is the Statue of Liberty's being made of metal a fact? This should be denied by those who deny that there are any disjunctive facts. ${ }^{9}$ A problem with such an austere view is that it may make it practically impossible to determine of anything whether it is indeed a fact. As the quote from Russell I mentioned in the introduction says, "nothing can be known to be simple". It might be just as hard to determine of something that it is not disjunctive as to determine that it is not conjunctive.

Nevertheless, many philosophers have mixed feelings about disjunctive facts. Russell [21, p. 215] is inclined to think that there are no disjunctive facts, but he also notes that the denial of disjunctive facts lead to certain difficulties. According to Fine [6, p.55] there are disjunctive propositional facts, but no disjunctive worldly facts. Armstrong [1, pp.43-46] denies the existence of disjunctive first-class facts, but he has no problem with disjunctive second-class and third-class facts, where these notions are defined in terms of classes of relations: first-class relations are universals; secondclass relations have the necessary and sufficient condition that when truly predicated of particulars, the resultant truth is a contingent one; third-class relations are relations that are not contingent (for example, being identical with a). Armstrong explicitly notes that he has no objection to admitting

\footnotetext{
${ }^{9}$ At least if being of metal means being of iron or being of copper or being of copper, steel and gold, etc.
} 
disjunctive and negative relations, provided they are at best second-class relations.

Although I do not use Armstrong's classification for states and relations, I assume that for all states 'out there' $s, s^{\prime}$ there exists a disjunctive state $s \otimes s^{\prime}$, and thus I define a disjunction $\Re \otimes \Re^{\prime}$ of relations analogously to the conjunction of relations. As an aside, I want to remark that, if desired, Armstrong's classification can be superimposed on the theory developed here.

The disjunction of relations is defined in a similar way as the conjunction of relations:

Definition 3.7 Let $\Re$ and $\Re^{\prime}$ be relations. Then, if it exists, the disjunction of $\Re$ and $\mathfrak{R}^{\prime}$ is defined as the relation induced by the disjunctive operation on states. It is denoted as $\Re \oslash \Re^{\prime}$, and its complexes as $\xi \oslash \xi^{\prime}$.

I postulate that the disjunction of any pair of relations is a relation as well:

P-12 Relation $\Re \oslash \Re^{\prime}$ exists for any relations $\mathfrak{R}, \Re^{\prime}$.

The next theorem also follows directly from Lemma 3.3.

Theorem 3.8 If disjunction of states is commutative (associative), then disjunction of complexes is commutative (associative) as well.

\subsubsection{Negation of Relations}

Russell once remarked [21, p. 211]: "I argued that there were negative facts, and it nearly produced a riot: the class would not hear of there being negative facts at all." I want to sidestep the discussion of whether there really are negative facts, but nevertheless admit for any state 'out there' $s$ the existence of the negation of $s$, denoted as $-s$. In general, $-s$ should not be conceived as a negative state, since $s$ itself may also seen as the negation of $-s$.

The definition of the negation of a relation is straightforward.

Definition 3.9 Let $\Re$ and $\Re^{\prime}$ be relations. Then, if it exists, the negation of $\Re$ is defined as the relation induced by the negation operation on states. It is denoted as $-\Re$, and its complexes as $-\xi$.

I postulate that the negation of any relation is a relation as well:

P-13 Relation $-\Re$ exists for any relation $\Re$.

The next theorem about the double negation of states and complexes also follows directly from Lemma 3.3. 
Theorem 3.10 If for all states $s$ in $\Re$ we have $--s=s$, then for all complexes $\xi$ in $\Re$ we also have $-\xi=\xi$.

Note that, by the Theorem 3.10, atomic relations may in a certain sense also be regarded as complex.

When we combine the logical operations, we get again by Lemma 3.3 other interesting identities, like De Morgan laws:

\section{Theorem 3.11}

1. If for all states $s$ in $\Re, s^{\prime}$ in $\Re^{\prime}$ we have $-\left(s \otimes s^{\prime}\right)=-s \otimes-s^{\prime}$, then for all complexes $\xi$ in $\Re, \xi^{\prime}$ in $\Re^{\prime}$ we also have $-\left(\xi \otimes \xi^{\prime}\right)=-\xi \otimes-\xi^{\prime}$.

2. If for all states $s$ in $\Re, s^{\prime}$ in $\Re^{\prime}$ we have $-\left(s \otimes s^{\prime}\right)=-s \otimes-s^{\prime}$, then for all complexes $\xi$ in $\Re, \xi^{\prime}$ in $\Re^{\prime}$ we also have $-\left(\xi \otimes \xi^{\prime}\right)=-\xi \otimes-\xi^{\prime}$.

\subsection{Restriction of Relations}

Consider the dyadic love relation with relational complexes of the form $x \rightarrow y$. Starting with this relation, we may construct a self-love relation as follows. (1) Select a relational complex of the original love relation with two occurrences of the same object, say Venus. (2) Merge these two occurrences to obtain a single one. (3) Define as relational complexes of the self-love relation the complexes that can be obtained via substitutions for this single occurrence.

In a similar way, we may define the relation of loving Venus. First, select a relational complex of the original love relation corresponding to a state of someone loving Venus. Then hide the occurrence of Venus, and define relational complexes with only one occurrence, namely an occurrence in the role of lover.

I call relations constructed in this way restrictions. For the love relation we have the following (classes of) restrictions:

1. For any object $a$, a monadic relation with relational complexes $a \gg y$.

2. For any object $b$, a monadic relation with relational complexes $x \rightarrow b$.

3. A monadic relation with relational complexes $x \leftrightarrow x$.

4. For any objects $a$ and $b$, a medadic relation with just one relational complex $a \stackrel{\gg}{\infty} .^{10}$

Note that from the conjunction of the love relation with itself it is possible to regain the love relation by merging corresponding occurrences in a complex $a \gg b \oplus a \rightarrow b$. (I presuppose here that for all love-states $s \otimes s=s$, and use the identity criterion P-9.)

\footnotetext{
${ }^{10}$ It is perhaps questionable whether we should regard relations of arity 0 as 'real' relations, but I see no compelling reason to exclude them.
} 
The approach for restricting the love relation may be generalized in a straightforward way to other relations:

Definition 3.12 Let $\Re$ be a relation, and $\xi$ be one of its complexes. Let $\mathcal{P}$ be a partition of a subset of $\mathrm{Oc}(\xi)$ such that each element of $\mathcal{P}$ contains only occurrences of the same object.

We say that a relation $\mathfrak{R}^{\prime}$ is a restriction induced by $\mathcal{P}$ if there is a complex $\xi^{\prime}$ in $\Re^{\prime}$ and a bijection $\tau: \mathcal{P} \rightarrow \operatorname{Oc}\left(\xi^{\prime}\right)$ such that

1. each $\alpha^{\prime} \in \operatorname{Oc}\left(\xi^{\prime}\right)$ and $\alpha \in \tau^{-1}\left(\alpha^{\prime}\right)$ are occurrences of the same object,

2. for each complex $\xi^{\prime \prime}$ of $\Re^{\prime}$ there is a $\sigma^{\prime}$ such that $\xi^{\prime} \cdot \sigma^{\prime}=\xi^{\prime \prime}$,

3. a substitution $\sigma^{\prime}$ in $\xi^{\prime}$ is defined iff the substitution $\sigma$ in $\xi$ is defined with

$$
\sigma(\alpha)= \begin{cases}\sigma^{\prime}\left(\alpha^{\prime}\right) \text { with } \alpha \in \tau^{-1}\left(\alpha^{\prime}\right) & \text { if such an } \alpha^{\prime} \text { exists } \\ \text { the object of } \alpha & \text { otherwise }\end{cases}
$$

and $\mathrm{S}\left(\xi^{\prime} \cdot \sigma^{\prime}\right)=\mathrm{S}(\xi \cdot \sigma)$.

Note that this operation does two things at once. It hides the occurrences not in $\bigcup \mathcal{P}$, and it merges the occurrences in each element of $\mathcal{P}$.

The identity criteria for relations and relational complexes guarantee that restrictions are thus uniquely defined:

Lemma 3.13 There is at most one relation a restriction induced by $\mathcal{P}$.

Proof Suppose that $\mathfrak{R}$ and $\mathfrak{R}^{\prime}$ are both restrictions induced by $\mathcal{P}$. Then for every complex in $\mathfrak{R}$ there is a qualitatively identical complex in $\mathfrak{R}^{\prime}$, and vice versa. So, by Lemma $2.8, \Re$ and $\Re^{\prime}$ are identical.

I postulate for restrictions the following principle:

P-14 Any restriction of a relation is also a relation.

Note that the definition of a restriction of relations allows us to choose $\mathcal{P}=\emptyset$. This gives a medadic relation with only one complex. Also note that if in a relation all complexes can be obtained from each other by a substitution, then choosing $\mathcal{P}=\{\{\alpha\} \mid \alpha \in \xi\}$ results in the original relation.

Although the definition of restrictions is simple, there are certain subtleties that need to be discussed. 
A state may have more corresponding relational complexes in a restriction than in the original relation:

Example 3.14 Let $\Re$ be the 'double' love relation with relational complexes of the form $x \gg y \otimes u \leftrightarrow v$. Then each state of this relation has one corresponding relational complex. But in the restriction $\Re^{\prime}$ obtained by merging the occurrences of $b$ in the relational complex $a \gg b \otimes b \leftrightarrow \otimes_{\rightarrow}$, the state corresponding with $a \gg b \otimes b \quad \gg \rightarrow a$ has $t w o$ corresponding relational complexes: one complex with one occurrence of $a$ and two of $b$ and another complex with two occurrences of $a$ and one of $b$. (See Fig. 2.)

A state may also have fewer corresponding relational complexes in a restriction than in the original relation:

Example 3.15 For the relation $\mathfrak{R}^{\prime}$ of Example 3.14, we can define a further restriction $\Re^{\prime \prime}$ by also merging the occurrences of $a$ in the relational complex corresponding to the state of $a \gg b \otimes b \rightarrow a$. This state has two relational complexes in $\mathfrak{R}^{\prime}$, but only one in $\mathfrak{R}^{\prime \prime}$, as follows from the definition of restrictions.

As we would expect of any robust theory of relations, restriction is a transitive operation:

Theorem 3.16 If $\Re^{\prime \prime}$ is a restriction of $\Re^{\prime}$ and $\Re^{\prime}$ is a restriction of $\Re$, then $\Re^{\prime \prime}$ is also a restriction of $\Re$.

Furthermore, if $\Re_{1}^{\prime}$ is a restriction of $\Re_{1}$ and $\Re_{2}^{\prime}$ is a restriction of $\Re_{2}$, then $\Re_{1}^{\prime} \otimes \Re_{2}^{\prime}, \Re_{1}^{\prime} \oslash \Re_{2}^{\prime},-\Re_{1}^{\prime}$ are restrictions of $\Re_{1} \otimes \Re_{2}, \Re_{1} \oslash \Re_{2}, \Re_{1}$, respectively.

Proof Let $\Re^{\prime}$ be the restriction of $\Re$ induced by $\mathcal{P}$, and let $\Re^{\prime \prime}$ be the restriction of $\mathfrak{R}^{\prime}$ induced by $\mathcal{P}^{\prime}$. Let $\tau: \mathcal{P} \rightarrow \mathrm{Oc}\left(\xi^{\prime}\right)$ be a bijection fulfilling the conditions of Definition 3.12. Now define $\widetilde{\Re}$ as the restriction of $\Re$ induced by

$$
\left\{\bigcup \tau^{-1}[x] \mid x \in \mathcal{P}^{\prime}\right\} .
$$

Since substitution is coalescence-free, it follows that $\widetilde{\Re}$ is qualitatively the same as $\mathfrak{R}^{\prime \prime}$. So, by P-9, the identity criterion for relations, it follows that they are identical.

The second part of the theorem can be proved in a similar way.
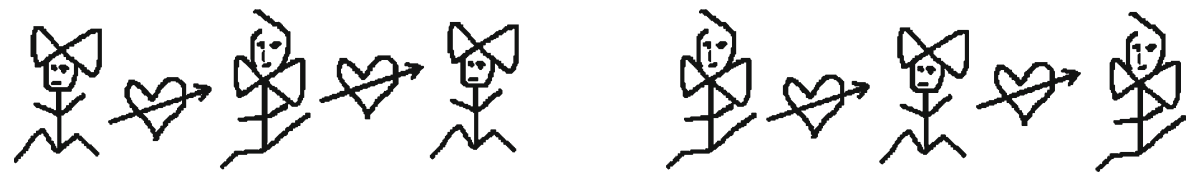

Fig. 2 Two complexes for the same state 
By the identity criterion for relations (Principle P-9), it follows that we can regain the relation $\Re$ by a restriction of $\Re \oplus \Re$ if each state of $\Re$ can be obtained from any other state via substitution.

Coordinating Occurrences By merging occurrences, the original occurrences are no longer present in the resulting relation. This would be different if we had a mechanism for linking occurrences. In his book 'Semantic Relationism' [8], Fine introduces the notion of coordination among certain entities as the strongest relation of synonymy. One of the forms of coordination concerns constituents of thought. In line with this, we might consider introducing coordination among occurrences of the same object in relational complexes. This would allow us to distinguish, for example, between (1) the relational complex of Cicero loving Cicero with two uncoordinated occurrences of Cicero, (2) the relational complex of Cicero loving Cicero with two coordinated occurrences of Cicero, and (3) the relational complex of Cicero loving himself with only one occurrence of Cicero. However, the applicability of coordination for elements of thought does not automatically mean that coordination is also needed on the level of relational complexes. I regard this as a topic for future investigation.

\section{Alternative Theories}

The polymorphic theory is of course not the only possible theory for relations. In this section we consider some alternative theories. In particular, we will take a look at theories that do not posit both states 'out there' and complexes.

\subsection{A Theory Without Complexes}

Can we build a theory with only states 'out there' and in which the states themselves have occurrences? The answer may depend on how refined the states are, but let us suppose that the states 'out there' are empirical entities. Then, as I will show, such a theory has unacceptable limitations.

There are basically two choices for a theory with only states:

(i) a theory in which each state belongs to only one relation;

(ii) a theory in which a state may belong to more than one relation.

The first option would accommodate only a very small class of relations. It would, for example, not be possible to define the conjunction and disjunction of a relation with itself, and also restrictions of a relation could not be defined, because these operations would yield states that were already in the original relations.

So, let us consider the more liberal kind of relational theory, where a state 'out there' may belong to more than one relation, and in which the states have occurrences relative to a relation. Equivalently, we may assume that relations do have complexes with occurrences, but no two complexes of the same relation correspond to the same state. This option gives considerably 
more freedom than the first one, but unfortunately, also this kind of theory has severe problems.

As follows from Example 2.2, a theory without complexes cannot accommodate a relation like the parthood relation if states are conceived of as possible worlds. Furthermore, for some relations the conjunction cannot be defined in such a theory. This is not only the case for conjunctions where $s \rrbracket \neg S$ and $s^{\prime} \oplus \neg s^{\prime}$ are taken as identical states, but also for some conjunctions without 'negative' components. We have already seen this in Example 2.3. There we considered the conjunction of the binary love relation with states $x \rightarrow y$, and the unary relation with states $x \rightarrow d$ with $d$ a fixed object. We showed that the composition principle for substitution (P-7) cannot be fulfilled if each state has only one corresponding complex in the conjunctive relation. The problem is that for the state $a \gg d \otimes c \rightarrow d$ the non-hidden occurrence of $d$ should on the one hand be 'part of' the conjunct $a \gg d$, and on the other of the conjunct $c \gg d$. This shortcoming shows that a theory with only (empirical) states 'out there' cannot give a fully adequate account of the logical structure of relations.

\subsection{A Theory Without States}

It might be argued that in the polymorphic theory, the states 'out there' play a rather modest role. They may be looked at as a means for classifying relational complexes. If we could develop a plausible theory of relations without states, then that theory might be the first choice.

There are two ways to go. We can try to develop a theory that makes use of an equivalence relation on complexes or we can try to develop a completely free-standing theory of relations without such an equivalence relation. Let us consider both options.

\subsubsection{A Theory with Empirically Indistinguishable Complexes}

We can define an equivalence relation $\approx$ on complexes, where $\xi \approx \xi^{\prime}$ means that $\xi$ and $\xi^{\prime}$ are empirically indistinguishable from each other, that is, necessarily $\xi$ obtains just in case $\xi^{\prime}$ obtains. With this equivalence relation, we can build a relational theory that makes no reference to states at all. Figure 3 depicts an entity-relationship (ER) diagram for this theory.

We can formulate principles for this state-less theory analogous to those of the polymorphic theory, with instead of P-2 a principle that says that each complex is empirically indistinguishable from one or more other complexes. Furthermore, some definitions need modifications, but most of them are straightforward. In particular, where $S(\xi)=S\left(\xi^{\prime}\right)$ is used, this should be replaced by $\xi \approx \xi^{\prime}$. Only for the logical operations the modifications are a bit less trivial. For example, in the definition of conjunction we should stipulate that necessarily $\xi \otimes \xi^{\prime}$ obtains iff $\xi$ obtains and $\xi^{\prime}$ obtains.

Apparently, we can develop a relational theory that is very similar to the polymorphic theory. The question is: which theory is to be preferred? 
Fig. 3 ER diagram for a relational theory without states

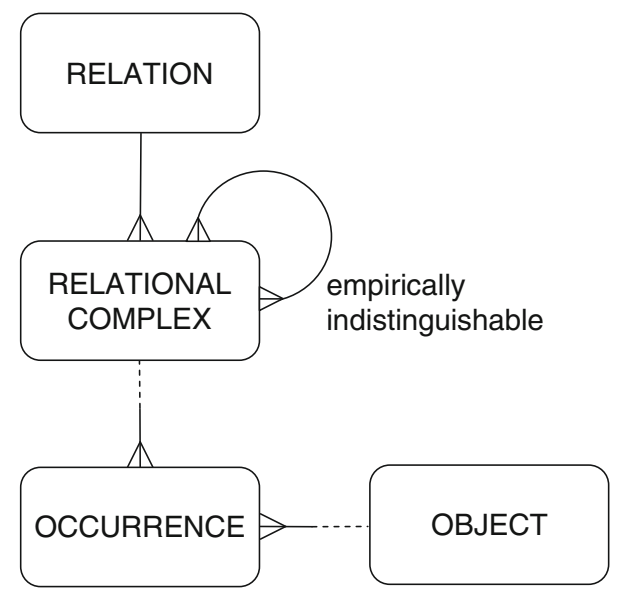

Unfortunately, I have no definite answer; there are strong arguments for both positions.

If states 'out there' are nothing but abstractions from complexes and there is no need for referencing them, then by the principle of parsimony, the stateless theory may be more appealing. However, if states are really 'out there' and complexes are structured perspectives on them, then the polymorphic theory seems more adequate. Or if the relational states are a proper subset of the states 'out there', then this would also be a consideration in favor of the polymorphic theory. The polymorphic theory is also more refined than the state-less theory if not all relational states are empirical entities. Furthermore, it may seem arbitrary to accept complexes in one's ontology, but not states.

\subsubsection{A Free-Standing Theory}

Suppose our objective is to develop a completely free-standing relational theory that neither refers to states nor makes use of the notion of empirical indistinguishability. Then we cannot postulate identity criteria for relations and relational complexes similar to the principles P-9 and P-10, because these principles make essential use of an equivalence between complexes. For the same reason, the operations on relations need to be redefined. Let us consider how this could be done.

Logical operations. Any definition of conjunction of relations should provide clear identity criteria for conjunctive complexes. Probably we want identities like $\xi \otimes \xi^{\prime}=\xi^{\prime} \otimes \xi$ and $\left(\xi \otimes \xi^{\prime}\right) \oplus \xi^{\prime \prime}=\xi \otimes\left(\xi^{\prime} \oplus \xi^{\prime \prime}\right)$, and possibly other identities as well (like $\xi \otimes \xi=\xi$ under certain conditions). We could perhaps give an axiomatic specification, but what do we have for its justification, and in what sense would such a specification be complete? If disjunction and negation are also taken into account, the challenge becomes even bigger. Then we have, for example, also to justify-again without referencing states or the like-whether or not $\xi \otimes \xi^{\prime}=-\left(-\xi \otimes-\xi^{\prime}\right)$. 
Restriction of relations. For restrictions we have to answer the following kind of questions:

1. Is the binary relation with complexes $\Re x y \oplus \Re x y$ the same as the binary relation with complexes $\Re x y$ ?

2. If $\Re$ is a transitive, binary relation, then is the ternary relation with complexes $\Re x y \oplus \Re y z \oplus \Re x z$ the same as the ternary relation with complexes $\Re x y \oplus \Re y z$ ?

3. For a restriction with complexes $\Re x x y$, when do we have $\Re x x y=\Re y y x$ ?

4. Is restriction of relations a transitive operation?

These questions do not necessarily have unique answers, because more than one restriction operation could perhaps be defined with different results. An important requirement for any definition, however, is that all resulting structures can be conceived of as genuine (complex) relations. Furthermore, we do not want a multiplicity of relations for essentially the same entity 'out there'. From this perspective, the preferred answer to each of the questions above would be "yes". But I do not see, what a general identity criterion for restrictions in a free-standing theory might be.

In conclusion, I don't think that a free-standing theory is a good alternative. What I regard as a key problem is how to understand the notion of complexes without the notion of states 'out there' or the notion of empirical indistinguishability. Furthermore, as the previous discussion suggests, we may end up with a complex and hard to justify set of identity rules.

\subsection{A Theory Without Occurrences of Objects}

For a theory without occurrences of objects, there are at least three options: (i) a theory with only states, (ii) a theory with only complexes, and (iii) a theory with states and complexes.

The first two options are not really worth considering here, since they have at least the same drawbacks as the theories discussed in Sections 4.1 and 4.2. The third option, however, seems more interesting.

We could define a simplified polymorphic theory with comparable principles as of the original theory, but with substitution working directly on objects. Equivalently, we could accept occurrences, but then all occurrences in a complex should be occurrences of different objects.

For the simplified polymorphic theory, we could define notions like the objects of a complex, the object-degree of a complex and the object-degree of $\mathcal{R}$ itself. Roughly stated, the objects of a complex are the objects for which it makes a difference for the resulting complex which objects are substituted for them. See $[15,17]$ for more detailed definitions.

To every relation-like structure $\breve{\mathfrak{R}}$ of the polymorphic theory there corresponds a unique structure $\mathcal{R}$ of the simplified polymorphic theory. The idea is 
to define for each complex $\xi$ in $\breve{\Re}$ and function $\delta: O \rightarrow O$, with $O$ the objects of $\Re$,

$$
\xi \odot \delta
$$

as the complex obtained by substituting in $\xi$ simultaneously $\delta(a)$ for each occurrence of each object $a$. The complexes of $\mathcal{R}$ may then be defined as equivalence classes of complexes of $\breve{\Re}$, where $\xi$ and $\xi^{\prime}$ are considered equivalent if $\mathrm{S}(\xi \odot \delta)=\mathrm{S}\left(\xi^{\prime} \odot \delta\right)$ for any $\delta: O \rightarrow O$.

An interesting question is under what conditions a relation-like structure $\breve{\Re}$ of the polymorphic theory can be uniquely reconstructed from a structure $\mathcal{R}$ of the occurrence-free theory. Let us look at a few simple cases.

1. Suppose that $\mathcal{R}$ has object-degree $n$, with $n$ finite. Then $\mathcal{R}$ has exactly one corresponding structure $\breve{\mathfrak{R}}$ of the same degree. Furthermore, $\breve{\Re}$ is the only corresponding structure of degree $\leq$ the number of objects of $\mathcal{R}$ with no dummy occurrences, where $\alpha$ is a dummy occurrence if $\mathbf{S}(\xi \cdot \sigma)=\mathbf{S}\left(\xi \cdot \sigma^{\prime}\right)$ for all substitutions $\sigma, \sigma^{\prime}$ with $\sigma=\mathrm{Oc}(\xi)-\{\alpha\} \sigma^{\prime}$.

2. Suppose again that $\mathcal{R}$ has object-degree $n$, with $n$ finite. Then $\mathcal{R}$ may have a corresponding structure $\breve{\Re}$ of degree > the number of objects of $\mathcal{R}$, and without dummy occurrences, as shown in the following examples:

(i) Let $\mathcal{R}_{0}$ be a structure with two objects and four complexes in the occurrence-free theory, and let $\mathcal{R}$ be the conjunction of $\mathcal{R}_{0}$ with itself. Then $\mathcal{R}$ may have more than one corresponding structure $\breve{\Re}$ of degree 4.

(ii) Let $\mathcal{R}$ have objects $a, b$, and complexes $\langle a, b\rangle,\langle b, a\rangle,\langle a\rangle,\langle b\rangle$ for states $s_{0}, s_{1}, s_{2}, s_{3}$, respectively. Then $\mathcal{R}$ has an initial complex, that is, a complex from which all other complexes can be obtained by substitution, but nevertheless $\mathcal{R}$ has a corresponding structure $\mathfrak{R}$ without dummy occurrences of fixed degree 3, namely a structure with two complexes for state $s_{0}$, two complexes for $s_{1}$, one complex for $s_{2}$, and one complex for $s_{3}$.

These examples show that the polymorphic theory is more powerful than the less refined theory without occurrences. The problem with the less refined theory is that the total number of objects of a relation may not be sufficient to express the interrelatedness of its complexes. In particular, certain complex relations cannot adequately be defined in it. This makes the polymorphic theory preferable from a metaphysical perspective.

\section{Conclusions}

Whether relations are really 'out there' or whether they are constructions of our mind may be hard to say, but in any case it is of metaphysical importance that they are a primary means for structuring the world. In this structuring, 
substituting objects for occurrences of other objects apparently plays a fundamental role. I find it difficult to imagine how to understand the world without the idea of substitution.

Different theories could be developed around the idea that substitution takes place in relational complexes. If complexes are conceived as structured perspectives on what there is 'out there', and in particular on relational states, then in principle the following views are possible for the correspondence between complexes and states:

View 1: Every relational state corresponds to exactly one relational complex.

View 2: Every relational state corresponds to one or more relational complexes, but to no more than one within a given relation.

View 3: Every relational state corresponds to one or more relational complexes, possibly even within the same relation.

The first view requires an ultra-refined notion of relational states or a very limited class of relations. Also for the second view the class of relations is seriously limited, among others because conjunction of relations might not always be defined (see Example 2.3). Moreover, in both the first and the second view, substitution might lead to a coalescence of occurrences for certain conjunctions of relations, which in some cases appears to be quite unnatural (see Example 3.5). I regard the third view as the most promising.

In this paper, I worked out a polymorphic theory of relations based on the third view in combination with a coalescence-free account of substitution. It is a very general theory in which no assumptions have been made about the existence of atomic facts or universals, nor any assumptions regarding the specific nature of relational states. In the remainder of this concluding section, I will highlight some of the distinguishing features of the theory.

Substitution works in such a way that it yields a one-to-one correspondence between the occurrences of the objects involved. A clear advantage of such a completely coalescence-free account of substitution is that relational complexes that can be obtained from each other by substitution have a uniform structure. For set-like relations, however, multiple occurrences of the same object in a complex may look a bit artificial. Although I see no objection in principle to making an exception for set-like relations by allowing for them a coalescence of occurrences, I have chosen not to do so since it would make the theory a bit more complicated.

In the polymorphic theory each complex corresponds to one state 'out there'. What complexes are, is completely determined by the network of substitutions between complexes and the corresponding states. This purely extensional view of complexes allows us to formulate a clear identity criterion for relations. Complementary to this criterion, we also have an identity criterion for relational complexes, where two complexes of a single relation are taken to be identical if substitutions in them are fully matching. These criteria enable us to define various operations on relations in a straightforward way in terms 
of operations on states, in particular conjunction, disjunction, negation and restrictions of relations.

Although the theory does not insist on a particular account of states 'out there', they most likely will be empirical entities. Nevertheless, mathematical relations can easily be represented in the theory. For let us suppose that states 'out there' are conceived as sets of possible worlds. Then a mathematical relation-modulo a certain equivalence-corresponds to the relational complexes of a relation (or of a relation-like structure) with two states, namely the set of all possible worlds and the empty set. (The equivalence is a consequence of the identity principles for relations and relational complexes. We get, for example, the same representation for the mathematical less than relation as for the greater than relation.) I am not sure whether any specific value should be attached to such representational possibilities of the polymorphic theory.

The principles of the polymorphic theory do not say which notions should be taken as primitive. They leave open the possibility that states 'out there' are merely abstractions from relational complexes. They do not even exclude the possibility that states are nothing but equivalence classes of relational complexes. But by conceiving of complexes as structured perspectives on relational states (as I did earlier), I certainly suggest that, in my view, states 'out there' are at least as primitive as complexes.

To the polymorphic theory of relations an objection could be raised that is superficially similar to an objection to what Fine [7] calls the standard view on relations. According to the standard view, arguments of a relation always come in a certain order. This implies that certain states 'out there' do not belong to a unique relation. But, according to Fine [7, pp.5-6], "we are much more inclined to suppose that there is a single underlying relation connecting the things together". Obviously, also for the polymorphic theory a state can belong to more than one relation. However, the non-uniqueness of the polymorphic theory is not objectionable in the same way. In the first place, in this theory the non-uniqueness is not a representational artifact, as is the case in the standard view. Secondly, the polymorphic theory does not exclude the existence of a class of basic relations for which the uniqueness of relational states applies.

It is good to contrast the polymorphic theory also to what Fine [7] calls the positionalist view on relations. In the positionalist view each relation comes with argument-places. An objection raised by Fine is that such a view cannot cope well with strictly symmetric relations, since switching objects assigned to argument-places would give a different complex. The polymorphic theory does not have this kind of problem. The identity criterion for relational complexes P-10 guarantees single relational complexes for the states of such relations.

There is a close relationship between the polymorphic theory and Fine's antipositionalist view on relations [7]. This is of course not very surprising, sinceas I said in Section 2.2-the substitution mechanism of the theory is directly based on the notion of substitution espoused by that view. The polymorphic theory could be regarded as an elaboration of the antipositionalist view.

An interesting issue is how the states and complexes in the polymorphic theory relate to Fregean thoughts. Perhaps disputes about the question 
whether thoughts are polymorphic stem from not making a proper distinction between states and complexes. It might not be a bad idea to divide Fregean thoughts into two types: one type corresponding to states 'out there' and one corresponding to relational complexes. Then the state of Willem-Alexander's loving Máxima could be identified with a single thought 'out there', and each of the numerous complexes of this state with a thought having a determinate structure.

The polymorphic theory of relations developed in this paper is quite detailed. Nevertheless, more principles may need to be introduced and some may require adjustments. A drastic departure that might be considered, would be to renounce any reference to states 'out there', and to use instead the notion of empirical indistinguishability for complexes. As discussed in Section 4.2, with this approach a very similar theory can be developed. The required modifications of the polymorphic theory are for the most part straightforward.

Should we choose a relational theory with states or one without states? For both options there seem to be strong arguments. For example, it seems natural to think of complexes as structured perspectives on states 'out there', and therefore to admit states as real entities. On the other hand, a theory with fewer entities is also appealing, although having fewer entities does not necessarily mean that the theory is simpler.

In conclusion, the ontological status of states 'out there' is controversial. But what I consider the main contribution of the analysis in this paper is that it shows that relational complexes and relational states are essentially different notions, and that taking the differences into account is necessary to understand the logical structure of relations.

Acknowledgements I am grateful to Kit Fine for his detailed comments and for asking to what extent states are required to develop a theory of logically complex relations. Furthermore, I thank Albert Visser, José Luis Bermúdez, Daniel Hill, and the referees for their comments on a manuscript of this paper.

Open Access This article is distributed under the terms of the Creative Commmons Attribution License which permits any use, distribution, and reproduction in any medium, provided the original author(s) and the source are credited.

\section{Appendix: Principles of the Polymorphic Theory}

\section{Structural Principles}

P-1 Each relation 'has' one or more relational complexes and each relational complex belongs to one and only one relation.

P-2 Each relational complex corresponds to one state 'out there' and each state 'out there' may correspond to one or more relational complexes.

P-3 Each relational complex may contain one or more occurrences of objects and each occurrence belongs to one and only one relational complex.

P-4 Each occurrence is the occurrence of one object and each object may be the content of one or more occurrences. 


\section{Substitution Principles}

P-5 Any substitution of objects for occurrences in a relational complex results in exactly one relational complex of the same relation.

P-6 For any complex, the identity substitution results in the same complex.

P-7 Composition principle: If a substitution $\sigma$ in a relational complex $\xi$ results in $\xi^{\prime}$, then there is a bijection $\mu$ from the occurrences in $\xi$ to the occurrences in $\xi^{\prime}$, such that

(a) $\mu$ maps each occurrence $\alpha$ in $\xi$ to an occurrence of the object substituted by $\sigma$ for $\alpha$,

(b) any substitution $\sigma^{\prime}$ in $\xi^{\prime}$ gives the same result as substituting in $\xi$ for each occurrence $\alpha$ the object that $\sigma^{\prime}$ substitutes for $\mu(\alpha)$.

(Or in symbols, $(\xi \cdot \sigma) \cdot \sigma^{\prime}=\xi \cdot\left(\mu \cdot \sigma^{\prime}\right)$.)

\section{Connectivity Principle}

P-8 For any relation $\Re$ of fixed finite degree, any relational complex of $\Re$ can be obtained from any other relational complex of $\Re$ via substitution.

\section{Identity Criteria}

P-9 Relations are identical if and only if they are qualitatively the same.

P-10 Relational complexes that belong to the same relation are identical if and only if they are qualitatively the same.

\section{Principles for Operations}

P-11 Relation $\Re \oplus \Re^{\prime}$ exists for any relations $\mathfrak{R}, \Re^{\prime}$.

P-12 Relation $\Re \oslash \Re^{\prime}$ exists for any relations $\Re, \Re^{\prime}$.

P-13 Relation $-\Re$ exists for any relation $\Re$.

P-14 Any restriction of a relation is also a relation.

\section{References}

1. Armstrong, D. (1997). A world of states of affairs. Cambridge: Cambridge University Press.

2. Barker, R. (1992). CASE*Method: entity relationship modelling. New York: Addison-Wesley Publishing Company.

3. Bell, D. (1996). The formation of concepts and the structure of thoughts. Philosophy and Phenomenological Research, LVI, 583-596.

4. Bermúdez, J. L. (2001). Frege on thoughts and their structure. Logical Analysis and History of Philosophy, 4, 87-105.

5. Bolzano, B. (1975). Paradoxien des Unendlichen. Hamburg: Felix Meiner Verlag.

6. Fine, K. (1982). First-order modal theories III-facts. Synthese, 53, 43-122.

7. Fine, K. (2000). Neutral relations. The Philosophical Review, 109, 1-33.

8. Fine, K. (2007). Semantic relationism. Oxford: Blackwell Publishing.

9. Frege, G. (1963). Compound thoughts. Trans. by R. H. Stoothoff. Mind, 72(285), pp. 1-17. 
10. Frege, G. (1983). Logik in der Mathematik. In H. Hermes, F. Kambartel, \& F. Kaulbach (Eds.), Nachgelassene Schriften (pp. 219-270). Hamburg: Felix Meiner Verlag.

11. Frege, G. (2000). Begriffsschrift. In J. van Heijenoort (Ed.), From Frege to Gödel: A Source Book in Mathematical Logic, 1879-1931 (pp. 5-82). New York: toExcel.

12. Frege, G. (2003). Der Gedanke-eine logische Untersuchung. In G. Patzig (Ed), Logische Untersuchungen (pp 35-62). Göttingen: Vandenhoeck and Ruprecht.

13. Frege, G. (2003). Logische Untersuchungen-Dritter Teil: Gedankengefüge. In G. Patzig (Ed.), Logische Untersuchungen (pp. 85-107). Göttingen: Vandenhoeck and Ruprecht.

14. Hodes, H. (1982). The composition of Fregean thoughts. Philosophical Studies, 41(2), 161-178.

15. Leo, J. (2008). Modeling relations. Journal of Philosophical Logic, 37, 353-385.

16. Leo, J. (2008). The identity of argument-places. The Review of Symbolic Logic, 1(3), 335-354.

17. Leo, J. (2010). Modeling occurrences of objects in relations. The Review of Symbolic Logic, $3(1), 145-174$.

18. Lewis, D. (2002). On the Plurality of Worlds. Oxford: Blackwell Publishers.

19. Linsky, B. (2003). The metaphysics of logical atomism. In N. Griffin (Ed.) The Cambridge companion to Bertrand Russell (pp. 371-391). Cambridge: Cambridge University Press.

20. MacBride, F. (2005). The particular-universal distinction: a dogma of metaphysics? Mind, 114, 565-614.

21. Russell, B. (1956). The philosophy of logical atomism. In R. C. Marsh (Ed.), Logic and knowledge, essays 1901-1950 (pp. 175-281). London: George Allen \& Unwin.

22. Russell, B. (1972). The principles of mathematics. London: George Allen \& Unwin.

23. Russell, B. (1984). Theory of knowledge. In E. R. Eames (Ed.), Theory of knowledge, the 1913 manuscript. London: Allen and Unwin.

24. Russell, B. (2005). My philosophical development. Routledge, New York.

25. Simons, P. (1992). The old problem of complex and fact. In J. T. J. Srzednicki (Ed.), Philosophy and logic in central Europe from Bolzano to Tarski: Selected essays (pp. 319-338). Dordrecht: Kluwer Academic Publishers.

26. Thalheim, B. (2000). Entity-relationship modeling: Foundations of database technology. Berlin: Springer.

27. Wittgenstein, L. (1975). Philosophical remarks. Oxford: Basil Blackwell.

28. Wittgenstein, L. (1984). Notebooks, 1914-1916. Oxford: Basil Blackwell. 